\title{
Vonoprazan Versus Lansoprazole in the Treatment of Artificial Gastric Ulcers After Endoscopic Submucosal Dissection: a Randomized Controlled Trial
}

\section{Daisuke Kawai}

Tsuyama Chuo Hospital

Ryuta Takenaka ( $\sim$ rtakenak@gmail.com )

Tsuyama Chuo Hospital https://orcid.org/0000-0001-7632-1474

Mikako Ishiguro

Tsuyama Chuo Hospital

Shotaro Okanoue

Tsuyama Chuo Hospital

\section{Tatsuhiro Gotoda}

Tsuyama Chuo Hospital

\section{Yoshiyasu Kono}

Tsuyama Chuo Hospital

\section{Koji Takemoto}

Tsuyama Chuo Hospital

Hirofumi Tsugeno

Tsuyama Chuo Hospital

Shigeatsu Fujiki

Tsuyama Chuo Hospital

Research article

Keywords: Vonoprazan, Lansoprazole, Postoperative bleeding, Endoscopic submucosal dissection, Gastric neoplasm

Posted Date: December 21st, 2020

DOl: https://doi.org/10.21203/rs.3.rs-131064/v1

License: (a) This work is licensed under a Creative Commons Attribution 4.0 International License. Read Full License 


\section{Abstract}

Background: Vonoprazan is more potent and longer acting than traditional proton pump inhibitor. Although vonoprazan is expected to be superior to proton pump inhibitor, its efficacy in the treatment of gastric ulcers following endoscopic submucosal dissection (ESD) is not fully understood. The aim of this study was to evaluate the effectiveness of vonoprazan in artificial ulcer healing following ESD.

Methods: Patients with gastric tumors were randomly assigned to the vonoprazan group (group $\mathrm{V}$ ) or lansoprazole group (group L) after ESD. Patients received intravenous lansoprazole $(30 \mathrm{mg})$ twice on the day of ESD. Thereafter, patients were treated with vonoprazan $(20 \mathrm{mg} /$ day $)$ in group $V$ or lansoprazole (30 mg/day) in group L. Esophagogastroduodenoscopy was performed 4 and 8 weeks after the ESD.

Results: A total of 168 patients were analyzed. The 4-week healing rate for artificial ulcer was not significantly higher in group $V$ versus group $L(17 / 85,20.0 \%$ vs. $14 / 83,16.9 \%$, respectively). In addition, there were no significant differences between the 4-week shrinkage rates between the two groups. Postoperative bleeding occurred in none of the patients in group $V$ and three in group L. One patient in group $\mathrm{V}$ presented delayed perforation 2 days after ESD.

Conclusions: Vonoprazan might not be superior to lansoprazole in the healing of artificial gastric ulcer after ESD.

Trial registration; University hospital Medical Information Network (registration number: UMIN000016642), Registered 27 February 2015, https://www.umin.ac.jp/ctr/index-j.htm

\section{Background}

Endoscopic submucosal dissection (ESD) for early gastric cancer has been widely accepted and is a wellestablished procedure in Eastern and Western countries [1-4]. ESD provides higher rate of en bloc and R0 resection rate, but is occasionally associated with some complications, such as bleeding and perforation [5]. Conventional proton pump inhibitors (PPIs) have been widely used for treating ESD-induced gastric ulcers. Although PPIs support to heal such lesions, some ulcers fail to heal; therefore, a more effective therapy is warranted.

Vonoprazan is a novel suppressant of gastric acid secretion and an active potassium-competitive acid blocker (P-CAB) [6]. Similar to PPIs, P-CABs inhibit gastric H+/K+-ATPase. Unlike PPIs, P-CABs inhibit the enzyme in a $\mathrm{K}+$-competitive and reversible manner. The inhibitory effect of vonoprazan on gastric acid secretion is largely unaffected by ambient $\mathrm{pH}$. Therefore, vonoprazan is more potent with a more longlasting effect than that of PPIs [7, 8]. Vonoprazan is expected to be superior to PPIs, and lead to earlier healing of ESD-induced gastric ulcers versus conventional PPI-based therapy. However, its efficacy in treating these ulcers remains unclear. Hence, in this study, we aimed to evaluate the effectiveness of vonoprazan in healing artificial ulcers after ESD. 


\section{Methods}

This study was a prospective, single-center, randomized controlled trial (RCT). The study protocol was approved by the local ethics committee of Tsuyama Chuo Hospital, Tsuyama, Japan and registered with the University hospital Medical Information Network (URL: https://www.umin.ac.jp/ctr/index-j.htm; registration number: UMIN000016642). Written informed consent was provided by each patient.

Patients with gastric tumors were enrolled between April 2015 and December 2017. The exclusion criteria were: remnant stomach, administration of antithrombotic agents, non-steroidal anti-inflammatory drugs and steroids; occurrence of complication during ESD; allergy to lansoprazole or vonoprazan; and unwillingness to participate in the study. Following ESD, patients were randomly assigned to the vonoprazan group (group $\mathrm{V}$ ) or the lansoprazole group (group $\mathrm{L}$ ) through the minimization method using Kullback-Leibler divergence [9]. The ESD ulcer index was used to balance continuous variables. On the day of ESD, patients received $30 \mathrm{mg}$ of lansoprazole twice intravenously. From postoperative day 2 , patients in groups $V$ and $L$ received $20 \mathrm{mg} /$ day of vonoprazan and $30 \mathrm{mg} /$ day of lansoprazole for 8 weeks, respectively.

Esophagogastroduodenoscopy was performed 4 and 8 weeks after the ESD (Fig. 1). During the follow-up endoscopy, the artificial ulcer was evaluated using a gastric ulcer stage system [10], and the length and width of the artificial ulcer were evaluated with measure forceps (M2-2C, M2-3U or M2-4K, Olympus Co, Japan) (Fig. 2A). The ulcer healing was defined as scarring at S1 or S2. The ESD ulcer index was calculated by multiplying the length by the width of the resected specimen. The 4- and 8-week ulcer indices were also calculated by multiplying the length by the width of the artificial ulcer at 4 and 8 weeks after ESD, respectively (Fig. 2B). The shrinking rate was defined as [1-(the ulcer index)/(the ESD ulcer index)] $\times 100(\%)$.

The primary endpoint was the healing rate of the artificial ulcer at 4 weeks after ESD. The secondary endpoints were: the healing rate at 8 weeks; shrinkage rates of the artificial ulcers at 4 and 8 weeks; and complications, such as post-operative bleeding and delayed perforation.

The healing rate of ulcers after the 4-week administration of PPIs was reported as $11-35 \%[11,12]$. According to our previous data, the healing rate after a 4-week administration of PPIs was $24 \%$. A $20 \%$ improvement observed following the administration of vonoprazan was considered clinically effective. It was estimated that 82 cases were required in each group to have a power of $80 \%$ for detection of a difference at an $a=0.05$ level of significance using Fisher's exact test. Assuming a study drop-out rate of approximately $10 \%, 90$ cases were enrolled in each group.

\section{Results}

Of the 263 patients who underwent gastric ESD from April 2015 to December 2017, 182 patients (90 in group $V$ and 92 in group $L$ ) were eligible to participate in the study. Fourteen patients were excluded during follow-up mainly because of complications or the need for additional surgery. Finally, 85 and 83 
patients were allocated to group V and group L, respectively (Fig. 3). The age, gender, status of Helicobacter pylori infection, tumor location, and ESD ulcer index of the two groups did not differ significantly (Table 1). The 4-week healing rate of artificial ulcers was not significantly higher in group $\mathrm{V}$ versus group $L(17 / 85,20.0 \%$ vs. $14 / 83,16.9 \%$, respectively). Furthermore, there was no significant difference between the 4-week shrinkage rates noted in groups $V$ and L. Postoperative bleeding was observed in three patients of group $L$ and none of the patients in group $V$. One patient in group $V$ presented delayed perforation 2 days after ESD (Table 2). 
Table 1

Clinical characteristics of the study participants

\begin{tabular}{|c|c|c|c|c|c|}
\hline \multirow[b]{2}{*}{ Age (years), median (range) } & \multicolumn{2}{|c|}{$\begin{array}{l}\text { Vonoprazan group } \\
n=85\end{array}$} & \multicolumn{2}{|c|}{$\begin{array}{l}\text { Lansoprazole group } \\
n=83\end{array}$} & \multirow{2}{*}{$\begin{array}{l}\text { P value } \\
0.96\end{array}$} \\
\hline & 73 & $(47-89)$ & 73 & $(33-90)$ & \\
\hline Gender, male, n (\%) & 63 & $(74)$ & 58 & (70) & 0.61 \\
\hline \multicolumn{6}{|l|}{ Habits } \\
\hline Smoking, n (\%) & 16 & (19) & 16 & (19) & 1.00 \\
\hline Alcohol, n (\%) & 36 & $(42)$ & 34 & $(41)$ & 0.88 \\
\hline \multicolumn{6}{|l|}{ Helicobacter pylori status } \\
\hline Positive, n (\%) & 30 & (35) & 31 & $(37)$ & 0.87 \\
\hline \multicolumn{6}{|l|}{ Location of lesion } \\
\hline Upper third, n (\%) & 9 & $(11)$ & 11 & $(13)$ & 0.28 \\
\hline Middle third, n (\%) & 35 & $(41)$ & 42 & $(51)$ & \\
\hline Lower third, n (\%) & 41 & $(48)$ & 30 & $(36)$ & \\
\hline Lesion size (mm), median (range) & 10 & $(2-54)$ & 10 & $(2-40)$ & 0.85 \\
\hline \multicolumn{6}{|l|}{ Histology } \\
\hline Adenoma, n (\%) & 13 & $(15)$ & 20 & $(24)$ & 0.17 \\
\hline Differentiated adenocarcinoma, n (\%) & 70 & $(82)$ & 58 & (70) & \\
\hline Undifferentiated, adenocarcinoma,n (\%) & 2 & $(2)$ & 3 & $(4)$ & \\
\hline${ }^{\dagger} \mathrm{NET}$ G1 & 0 & $(0)$ & 2 & $(2)$ & \\
\hline \multicolumn{6}{|l|}{ Depth of invasion $(n=133)$} \\
\hline Mucosa & 66 & $(92)$ & 55 & $(90)$ & 0.77 \\
\hline Submucosa & 6 & (8) & 6 & $(10)$ & \\
\hline Ulcer scar in tumor, $\mathrm{n}(\%)$ & 6 & (7) & 7 & (8) & 0.78 \\
\hline${ }^{\ddagger}$ ESD ulcer index $\left(\mathrm{mm}^{2}\right)$, median (range) & 875 & $(260-4,000)$ & 875 & $(238-4,180)$ & 0.59 \\
\hline
\end{tabular}


Table 2

Clinical outcomes of the endoscopic submucosal dissection induced ulcers

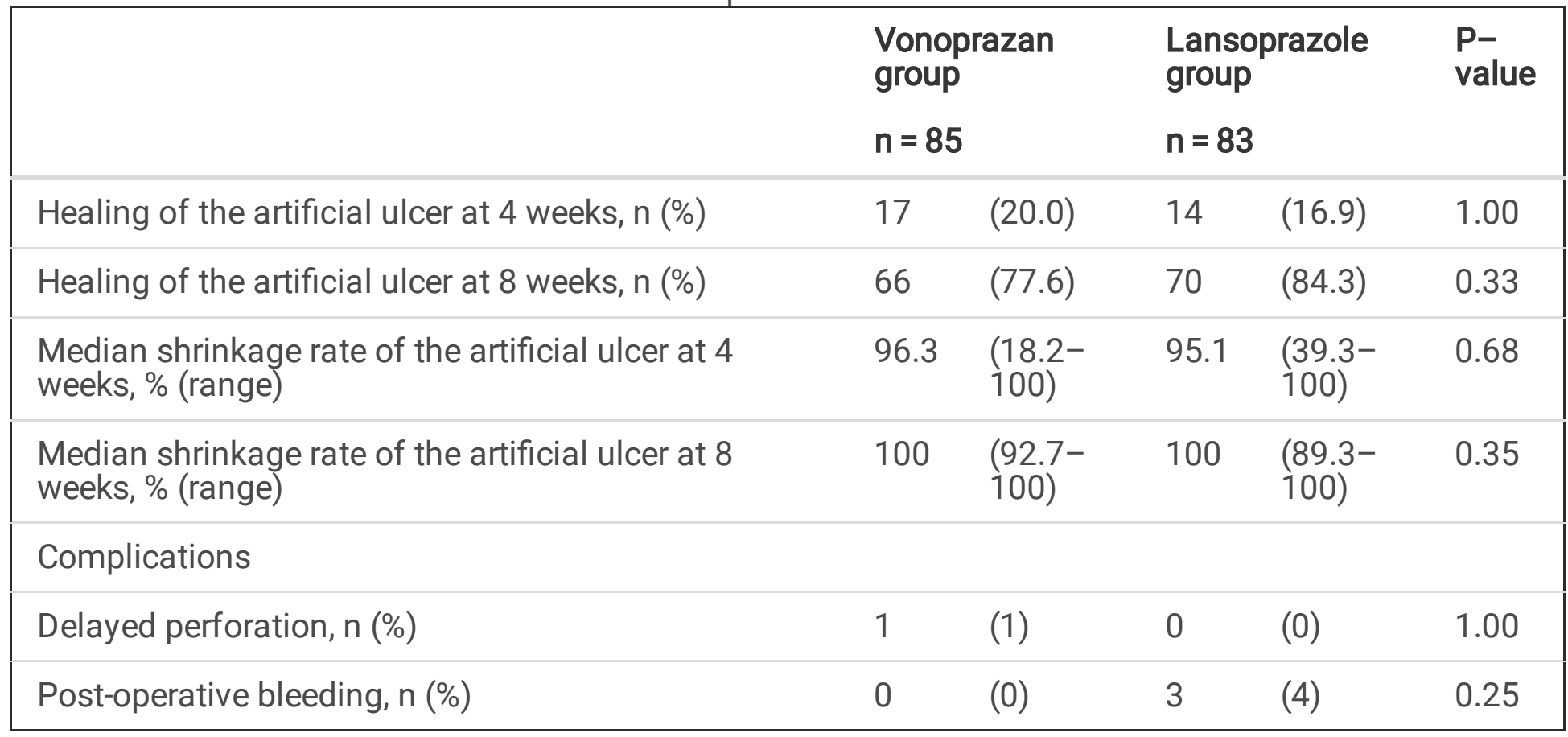

\section{Discussion}

In this RCT, we attempted to prove the superiority of vonoprazan to lansoprazole in the healing rate after gastric ESD in patients stratified according to the ESD ulcer index. Unfortunately, vonoprazan was not superior to lansoprazole in terms of the healing rates and shrinkage rates of artificial gastric ulcers at 4 and 8 weeks after ESD. Furthermore, there were no significant differences in the occurrence of postoperative bleeding and delayed perforation between the two groups.

Some RCTs suggested that vonoprazan was as effective as PPIs in the treatment of ESD-induced ulcer [13-15]. Our results were consistent with those previously reported, in which the healing rates at 4 weeks in the vonoprazan groups ranged $7.4-20.9 \%$. Our hypothesis was that the strong and rapid inhibition of gastric acid secretion by vonoprazan may enhance the healing of artificial ulcers. However, the effect of ulcer shrinkage in the vonoprazan group was similar to that noted in the PPI group. There may be two reasons for this result. Firstly, acid suppression by both vonoprazan and lansoprazole are excellent at shrinking artificial ulcers. Secondly, other factors than acid suppression are involved in the rapid resolution of ulcer. These factors include the existence of ulcer scar, ulcer area, ulcer site, blood coagulation status, Helicobacter pylori infection, and other comorbidities.

On the other hand, some studies concluded that vonoprazan was superior to PPIs for healing ESDinduced ulcers [16-19]. However, there were few prospective, randomized controlled studies conducted. Tsuchiya et al. reported that the vonoprazan group had a significantly superior shrinkage rate at 8 weeks; however, there was no significant difference observed in the rate of postoperative bleeding [16]. In this prospective study, the shrinkage rates until 6 weeks were not significantly different. However, the 8-week shrinkage rate was significantly higher in the vonoprazan group versus the PPI group. In a systematic 
review and network meta-analysis, the effect of vonoprazan at 8 weeks was superior to that of PPIs for the treatment of artificial ulcers following ESD [20]. However, another meta-analysis reported that the healing rate at 8 weeks was significantly higher in the PPI group versus the vonoprazan group [21]. Thus, the effect of vonoprazan remains controversial and further RCTs are warranted. Although the primary endpoint did not meet, we believe the findings of our study are meaningful.

In this investigation, only three patients in the PPI group developed postoperative bleeding. In the 5 RCTs (including this trial) conducted thus far, the rate of postoperative bleeding in the vonoprazan group range $0-5.4 \%$. Although the backgrounds of the study differ, the postoperative bleeding rate was equal or lower in the vonoprazan group versus the PPI group in all studies. Hamada et al. showed that vonoprazan efficaciously reduced the delayed bleeding rate in patients with an ESD-induced gastric ulcer in comparison with the threshold rate recorded using binomial testing [22]. A larger-scale study with postoperative bleeding as its primary endpoint or meta-analysis using RCTs may prove the efficacy of vonoprazan against this complication.

There were some limitations in this study. Firstly, this study was conducted in a single center in Japan and sample size was small. Secondly, patients receiving antithrombotic agents were excluded. As postoperative bleeding was associated with administration of antithrombotic agents, its rate may be underestimated in this study.

\section{Conclusions}

Vonoprazan was not superior to lansoprazole in the healing of artificial gastric ulcers after ESD. However, our data are meaningful because the effect of vonoprazan remains controversial and a larger-scale RCTs are required to verify the present findings.

\section{List Of Abbreviations}

ESD; endoscopic submucosal dissection

NET; neuroendocrine tumor

P-CAB; potassium-competitive acid blocker

PPl; proton pump inhibitor

RCT; randomized controlled trial

\section{Declarations}

\section{Ethics approval and consent to participate:}


The study was approved by the institutional review board of our hospital (Tsuyama Jihukai Ethics Committee) on March 3, 2015 (reference number 220) and was conducted in accordance with the Declaration of Helsinki. Written informed consent was provided by all patients.

\section{Consent for publication:}

Not applicable

\section{Availability of data and materials}

All data generated or analyzed during this study are available from the corresponding author on reasonable request.

\section{Competing interests:}

The authors declare that they have no competing interests.

\section{Funding}

There was no financial support provided for this study.

\section{Authors' contributions:}

Concept and design: DK and RT; data collection: DK, RT, MI, SO, TG, YK, KT, HT; analysis and interpretation of data: DK, RT and Ml; drafting of the article: DK and RT; critical revision of the article: SF. All authors read and approved the manuscript.

\section{Acknowledgements:}

We are grateful to Mr. Ryoichi Muraoka for supporting the dynamic randomization using Kullback-Leibler divergence.

\section{References}

1. Ono H, Kondo H, Gotoda T, Shirao K, Yamaguchi H, Saito D, et al. Endoscopic mucosal resection for treatment of early gastric cancer. Gut. 2001;48:225-9.

2. Fujishiro M, Yahagi N, Nakamura M, Kakushima N, Kodashima S, Ono S, et al. Successful outcomes of a novel endoscopic treatment for GI tumors: endoscopic submucosal dissection with a mixture of high-molecular-weight hyaluronic acid, glycerin, and sugar. Gastrointest Endosc. 2006;63:243-9.

3. Chung IK, Lee JH, Lee SH, Kim SJ, Cho JY, Cho WY, et al. Therapeutic outcomes in 1000 cases of endoscopic submucosal dissection for early gastric neoplasms: Korean ESD Study Group multicenter study. Gastrointest Endosc. 2009;69:1228-35.

4. Probst A, Pommer B, Golger D, Anthuber M, Arnholdt H, Messmann H. Endoscopic submucosal dissection in gastric neoplasia - experience from a European center. Endoscopy. 2010;42:1037-44. 
5. Saito I, Tsuji Y, Sakaguchi Y, Niimi K, Ono S, Kodashima S, et al. Complications related to gastric endoscopic submucosal dissection and their managements. Clin Endosc. 2014;47:398-403.

6. Shin JM, Inatomi N, Munson K, Munson K, Strugatsky D, Tokhtaeva E, et al. Characterization of a novel potassium-competitive acid blocker of the gastric H,K-ATPase, 1-[5-(2-fluorophenyl)-1-(pyridin3-ylsulfonyl)-1H-pyrrol-3-yl]-N-methylmethanamine monofumarate (TAK-438). J Pharmacol Exp Ther. 2011;339:412-20.

7. Hori Y, Imanishi A, Matsukawa J, Tsukimi Y, Nishida H, Arikawa Y, et al. 1-[5-(2-Fluorophenyl)-1(pyridin-3-ylsulfonyl)-1 H-pyrrol-3-yl]-N-methylmethanamine monofumarate (TAK-438), a novel and potent potassium-competitive acid blocker for the treatment of acid-related diseases. J Pharmacol Exp Ther. 201;335:231-8.

8. Matsukawa J, Hori Y, Nishida H, Kajino M, Inatomi N. A comparative study on the modes of action of TAK-438, a novel potassium-competitive acid blocker, and lansoprazole in primary cultured rabbit gastric glands. Biochem Pharmacol. 2011;81:1145-51.

9. Endo A, Nagatani F, Hamada C, Yoshimura I. Minimization method for balancing continuous prognostic variables between treatment and control groups using Kullback-Leibler divergence. Contemp Clin Trials. 2006;27:420-31.

10. Sakita T, Fukutomi H. Endoscopic diagnosis. In: Ulcer of the Stomach and Duodenum. Yoshitoshi Y, eds. Tokyo:Nankodo; 1971:198-208.

11. Asakuma Y, Kudo M, Matsui S, Okada M, Kawasaki M, Umehara Y, et al. Comparison of an ecabet sodium and proton pump inhibitor (PPI) combination therapy with PPI alone in the treatment of endoscopic submucosal dissection (ESD)-induced ulcers in early gastric cancer: prospective randomized study. Hepatogastroenterology. 2009;56:1270-3.

12. Kato T, Araki H, Onogi F, Ibuka T, Sugiyama A, Tomita E, et al. Clinical trial: rebamipide promotes gastric ulcer healing by proton pump inhibitor after endoscopic submucosal dissection--a randomized controlled study. J Gastroenterol. 2010;45:285-90.

13. Hirai A, Takeuchi T, Takahashi Y, Kawaguchi S, Ota K, Harada S,et al. Comparison of the Effects of Vonoprazan and Lansoprazole for Treating Endoscopic Submucosal Dissection-Induced Artificial Ulcers. Dig Dis Sci. 2018;63:974-81.

14. Ichida T, Ueyama S, Eto T, Kusano F, Sakai Y. Randomized Controlled Trial Comparing the Effects of Vonoprazan Plus Rebamipide and Esomeprazole Plus Rebamipide on Gastric Ulcer Healing Induced by Endoscopic Submucosal Dissection. Intern Med. 2019;58:159-66.

15. Ishii $Y$, Yamada H, Sato T, Sue S, Kaneko H, Irie K, et al. Effects of Vonoprazan Compared with Esomeprazole on the Healing of Artificial Postendoscopic Submucosal Dissection Ulcers: A Prospective, Multicenter, Two-Arm, Randomized Controlled Trial. Gastroenterol Res Pract. 2018:1615092.

16. Tsuchiya I, Kato Y, Tanida E, Masui Y, Kato S, Nakajima A, et al. Effect of vonoprazan on the treatment of artificial gastric ulcers after endoscopic submucosal dissection: Prospective randomized controlled trial. Dig Endosc. 2017;29:576-83. 
17. Maruoka D, Arai M, Kasamatsu S, Ishigami H, Taida T, Okimoto $K$, et al. Vonoprazan is superior to proton pump inhibitors in healing artificial ulcers of the stomach post-endoscopic submucosal dissection: A propensity score-matching analysis. Dig Endosc. 2017;29:57-64.

18. Horikawa Y, Mizutamari H, Mimori N, Kato Y, Fushimi S, Sato S, et al. Short-term efficacy of potassium-competitive acid blocker following gastric endoscopic submucosal dissection: a propensity score analysis. Scand J Gastroenterol. 2018;53:243-51.

19. Yamasaki A, Yoshio T, Muramatsu Y, Horiuchi Y, Ishiyama A, Hirasawa T, et al. Vonoprazan is Superior to Rabeprazole for Healing Endoscopic Submucosal Dissection: Induced Ulcers. Digestion. 2018;97:170-6.

20. Kim EH, Park SW, Nam E, Lee JG, Park CH. Comparative efficacy of various anti-ulcer medications after gastric endoscopic submucosal dissection: a systematic review and network meta-analysis. Surg Endosc. 2019;33:1271-83.

21. Kang H, Kim BJ, Choi G, Kim JG. Vonoprazan versus proton pump inhibitors for the management of gastric endoscopic submucosal dissection-induced artificial ulcer: A systematic review with metaanalysis. Medicine (Baltimore). 2019;98:e15860.

22. Hamada K, Uedo N, Tonai Y, Arao M, Suzuki S, Iwatsubo T, et al. Efficacy of vonoprazan in prevention of bleeding from endoscopic submucosal dissection-induced gastric ulcers: a prospective randomized phase II study. J Gastroenterol. 2019;54:122-30.

\section{Figures}




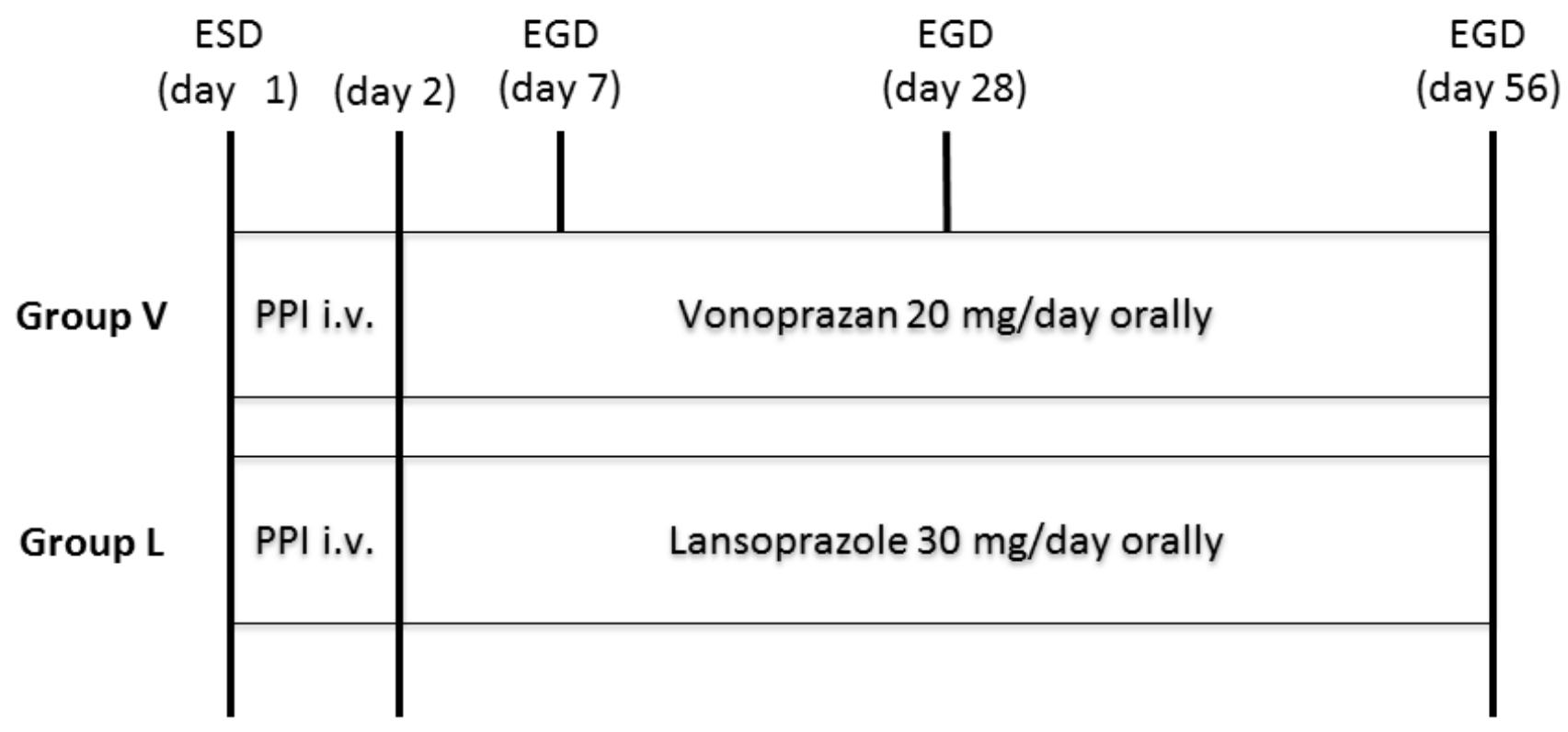

\section{Figure 1}

Study protocol. ESD, endoscopic submucosal dissection; EGD, esophagogastroduodenoscopy; group V, vonoprazan group; group L, lansoprazole; PPI, proton pump inhibitor; i.v., intravenous injection Flow chart of patients. ESD, endoscopic submucosal dissection 
(B)

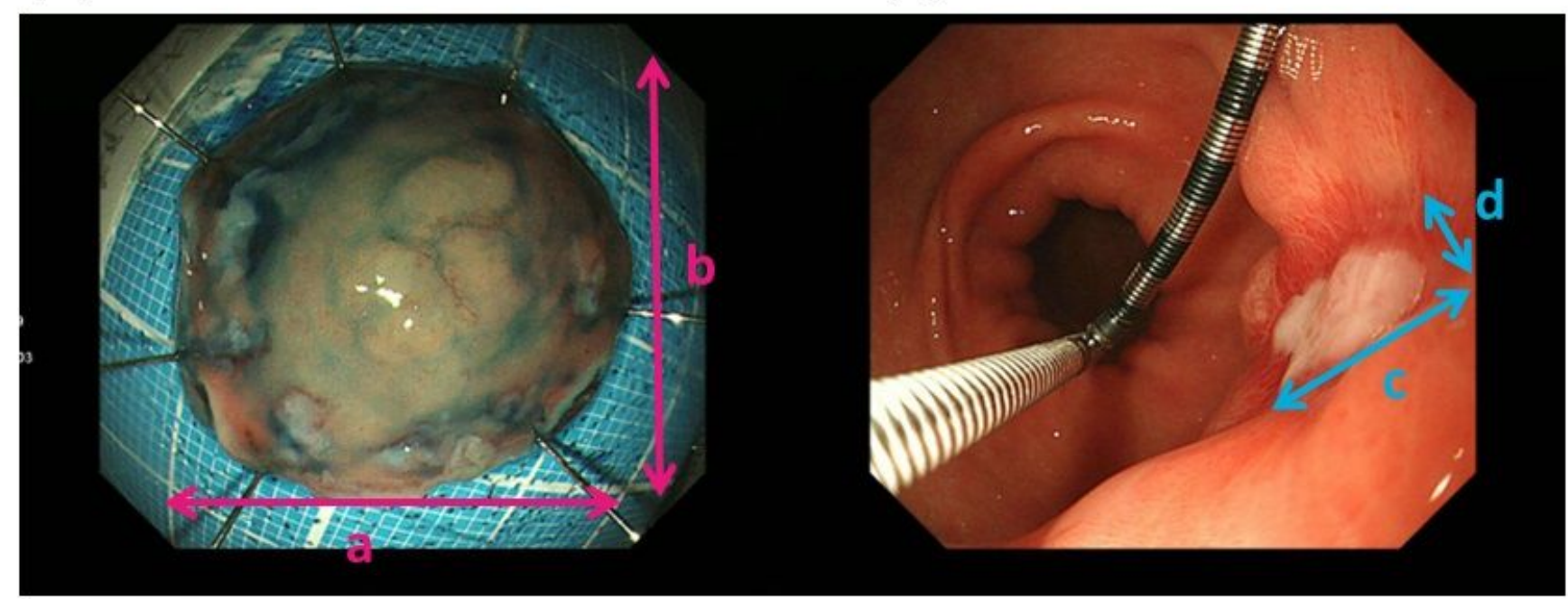

\section{Figure 2}

(A) The ESD ulcer index is calculated by multiplying the length (a) by the width (b) of the resected specimen. (B) The 4- and 8-week ulcer indices were calculated by multiplying the length (c) by the width (d) of the artificial ulcer at 4 and 8 weeks, respectively. 


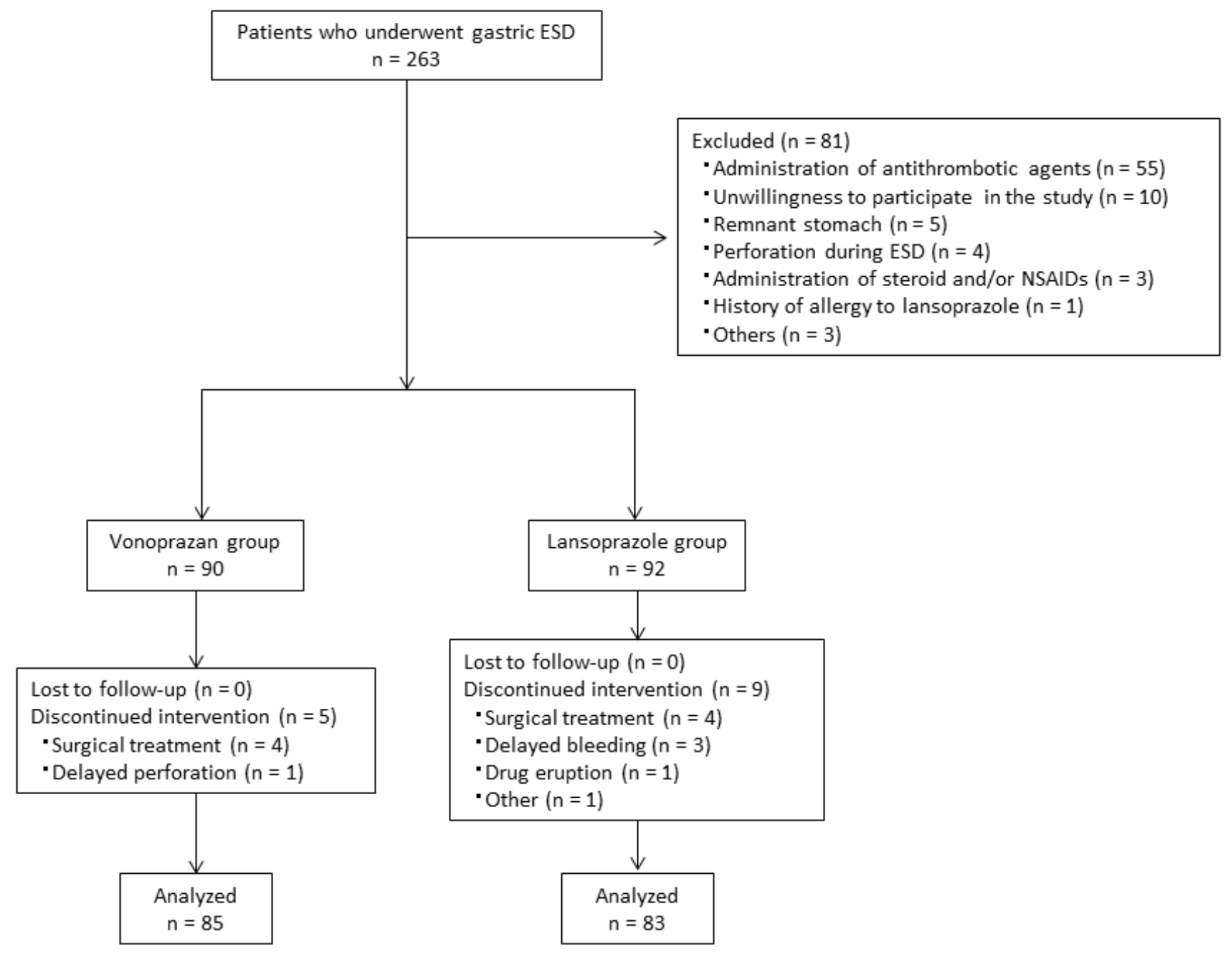

Figure 3

Flow chart of patients. ESD, endoscopic submucosal dissection

\section{Supplementary Files}

This is a list of supplementary files associated with this preprint. Click to download.

- CONSORT2010Checklist.doc 